\title{
El sexenio de investigación en Contabilidad
}

\section{The research assessment (sexenio) of accounting teaching staff in Spain}

\author{
José Antonio Cavero Rubio (cavero@umh.es) \\ Victoria Ferrández Serrano (v.ferrandez@umh.es)
}

Universidad Miguel Hernández de Elche (España)

http://dx.doi.org/10.12795/EDUCADE.2013.i04.02

\begin{abstract}
RESUMEN: El objetivo inicial atribuido a la concesión de los tramos de investigación, los denominados sexenios, era reconocer la excelencia investigadora de los profesores universitarios. Sin embargo, la normativa ha ido vinculando su posesión a una serie de facetas universitarias que limitan diferentes áreas de la actividad del profesorado universitario. El sexenio condiciona la retribución, los créditos docentes a impartir, la promoción, los estudios de doctorado, la participación en los comités asesores de la Comisión Nacional Evaluadora de la Actividad Investigadora (CNEAl), así como formar parte de las comisiones de acreditación y acceso a los cuerpos docentes universitarios. Dada la importancia que ha adquirido y adquirirá en un futuro poseer un tramo de investigación, en el presente trabajo se estudiarán las diferencias que existen en el porcentaje de profesores con sexenio, no sólo entre campos científicos, sino también entre áreas pertenecientes a un mismo campo, mostrando un interesante resultado en las diferencias por áreas. Nuestra condición de profesores universitarios de contabilidad, hace que centremos el presente estudio en el campo 8, donde se ubica el área de conocimiento "Economía Financiera y Contabilidad".

El debate que se plantea de los resultados de este trabajo es: (I) cómo un área de conocimiento, con el peso que Economía Financiera y Contabilidad tiene dentro de las Ciencias Económicas y Empresariales, tiene tan pocos representantes en los comités asesores que conceden los sexenios, y (II) hasta qué punto esta mínima representación conlleva unos peores resultados en la evaluación de los profesores del área, en la medida en que no hay expertos en la materia que evalúen atendiendo a las características particulares del área. Esto termina cerrando un círculo vicioso, ya que si no hay profesores con sexenios no pueden formar parte de las comisiones de expertos que los otorgan.
\end{abstract}

PALABRAS CLAVE: Profesores Universitarios, Sexenio, Investigación, Docencia, Teorías de Motivación.

\begin{abstract}
The initial objective attributed to the research assessment granting of university teaching staff (the so-called sexenio) was to recognize research effort of the university professors. However, this assessment has been linked to numerous aspects university life: determines the remuneration, teaching load, the possibility of promotion, the participation as teacher in masters and doctoral courses, the possibility of being examiner or even supervisor of doctoral dissertations, participation in the Advisory Committees of the National Commission for Evaluating the Research Activity (CNEAl), as well as being a part of the accreditation commissions and a long etcetera. Given the importance that this evaluation has gained and will acquire in a future, in the present work we will study the differences in the percentage of teachers with sexenio. Not only between scientific fields, but also between areas belonging to the same field, showing an interesting result in the differences by areas. Our condition of university accounting teachers, makes us to focus in the field 8, where the knowledge area of "Accounting and finance" is located.
\end{abstract}

Nota de investigación. Recibido: 22-01-13 - Versión revisada: 11-03-13, 21-03-13, Aceptado: 01-04-13

Licencia Creative Commons BY NC ND · $2013 \cdot$ Asociación Española de Contabilidad y Administración de Empresas - AECA 
The debate that arises from the results of this work is: Why Accounting and Finance has Sciences has so few representatives within the advisory committees in comparison with the importance in terms of teaching staff, students, etc.? Moreover, to what extent this minimal representation implies worse results in the evaluation of teachers of the area? This situation conforms a vicious circle: if there are no accounting teachers awarded with sexenio, they would not be able to be part of the Expert Commissions that grant those assessments.

KEYWORDS: Research assessment

\section{INTRODUCCIÓN}

La primera referencia legislativa a la evaluación del profesorado universitario en nuestro país se encuentra en la Ley de Reforma Universitaria de 25 de agosto de 1983, estableciendo que los Estatutos de la Universidad dispondrán los procedimientos para la evaluación periódica del rendimiento docente y científico del profesorado universitario. Posteriormente, el Real Decreto 1086/1989 transfiere al Estado, concretamente a la Comisión Nacional Evaluadora de la Actividad Investigadora (CNEAl), la potestad de evaluar cada seis años la actividad investigadora de su profesorado universitario, concediéndose un complemento retributivo adicional de productividad, el conocido "sexenio".

Sin embargo, la normativa universitaria está convirtiendo el sexenio en un requisito imprescindible para que el profesorado universitario pueda promocionar y realizar sus actividades docentes, investigadoras y de gestión, pasando de ser únicamente un incentivo que inicialmente premiaba económicamente la actividad investigadora, a ser considerado como un indicador de calidad. Mencionar a este respecto, que para realizar la evaluación de la investigación universitaria la CNEAl contará con el asesoramiento de comités formados por profesores con al menos tres sexenios.

Desde la perspectiva de la promoción del profesorado, la Ley Orgánica 6/2001 de Universidades, estableció un sistema para la promoción del profesorado universitario en dos fases, la habilitación y el acceso, siendo requisito para poder formar partes de ambas comisiones que los Titulares de Universidad (TU) y Catedráticos de Universidad (CU) tuviesen reconocido, al menos, un período de actividad investigadora, ampliándose a dos períodos para los CU si se trata de comisiones para CU. Posteriormente, la entrada en vigor de la Ley Orgánica 4/2007, sustituyó la habilitación por la acreditación nacional favorable de la ANECA, contando para ello con comisiones donde los CU y TU debían tener, al menos, tres y dos sexenios, respectivamente.

En el ámbito docente, el Real Decreto 99/2011, estableció que el coordinador del programa de doctorado debe tener reconocidos, al menos, dos sexenios y que el director de una escuela de doctorado debe poseer, al menos, tres. Y más recientemente, el Real Decreto-Ley 14/2012, de 20 de abril, de medidas urgentes de racionalización del gasto público en el ámbito educativo, donde no tener reconocidos sexenios penaliza al profesor aumentando su carga docente, aspecto que ha supuesto un incremento generalizado en el número de horas lectivas del profesorado, repercutiendo a su vez, de forma negativa en la actividad investigadora.

Esta situación tiende a acentuarse en el futuro, si tomamos en consideración la propuesta del Borrador del Estatuto del PDI de las Universidades Españolas (2011) donde el sexenio tendrá vinculación con la carrera del profesorado y con su retribución. Y más recientemente el Informe con las propuestas efectuadas por una comisión de expertos nombrados por el Consejo de Ministros, para la reforma y mejora 
de la calidad y eficiencia del sistema universitario español, el cual otorga al sexenio una importancia capital en las universidades: promoción, ocupar cargos académicos, gobernanza, contratación, selección, retribución, actividad investigadora, dedicación docente, financiación, prestigio y calidad de la universidad.

Como se ha puesto de manifiesto, el sexenio de investigación es cada vez más valorado, no tanto por el complemento retributivo que supone, sino por haberse convertido en un indicador de calidad y de reconocimiento, siendo un requisito imprescindible para poder abarcar todas las facetas universitarias. Aunque hay que reconocer que el establecimiento de este sistema de evaluación científica ha generado un incremento de la producción científica española o cuando menos ha propiciado su internacionalización y visibilidad (Sanz, 1995; Jiménez et al., 2003), no está exento de polémica, debatiéndose desde entonces la selección, adecuación, aplicación e influencia en las diferentes áreas de conocimiento (De las Heras y Polo, 2009; Delgado, 2009; Jiménez et al., 2011), y su repercusión sobre las diferencias existentes entre los distintos campos científicos y áreas de conocimiento.

Lo que se nos plantea es que si tanto valor tiene y tanta importancia se le otorga actualmente al sexenio de investigación, debería regularse mejor su reconocimiento y concesión, así como ajustar los criterios de evaluación de la investigación a las características particulares de cada área de conocimiento.

El protagonismo que está adquiriendo el sexenio de investigación delimita un nuevo escenario que es preciso analizar detenidamente. Con el presente trabajo se pretende reflexionar sobre porqué determinadas áreas de conocimiento presentan un porcentaje tan bajo de profesores con sexenios. Tomando como referencia los profesores TU y CU que tienen los sexenios reconocidos para participar en las comisiones de acceso a los cuerpos docentes universitarios, se analizará si existe correlación significativa con el número de presencias que los profesores de estas áreas han tenido en los comités asesores de la CNEAl encargados de reconocer los sexenios, prestando una especial atención al campo 8 "Ciencias Económicas y Empresariales" y, más concretamente, a la disciplina de contabilidad. Seguidamente, dado el escenario planteado, se analizará a la luz de las Teorías de motivación los efectos que las resoluciones de las citadas comisiones pueden tener sobre los profesores cuyos méritos investigadores son evaluados.

\section{LA EVALUACIÓN DE LA ACTIVIDAD INVESTIGADORA DEL PROFESORADO UNIVERSITARIO}

La evaluación de la investigación es un tema de gran actualidad y con una influencia cada vez más determinante sobre la vida académica, investigadora y profesional de individuos e incluso instituciones. En consecuencia, no es de extrañar que, especialmente desde la década de los ochenta, sean varios los autores que han decidido analizar diversos aspectos relacionados con la evaluación de la actividad investigadora (Dahllof et al., 1991; Vidal, 1993; Méndez et al., 1997; Cole, 1998; Campanario, 2002). A su vez, y siguiendo la tendencia general, la evaluación de la investigación en el ámbito de la economía también está siendo objeto de una notable atención por diversos autores. De esta forma, resultan muy interesantes los trabajos publicados en los últimos tiempos por autores como Coupé (2000), Bauwens et al. (2002), Combes y Linnemer (2002), Arnold et al. (2003), Dolado et al. (2001), Bergantiños et al. (2002) y Villar (2004).

A pesar de que la investigación es una actividad internacional, existe una gran divergencia en cuanto a los objetivos, consecuencias, prácticas y criterios a seguir en la evaluación de la investigación desarrollada en cada país (Sanz, 1995). Como se muestra en la Tabla 1, en España, la CNEAl articula la evaluación de la actividad 
investigadora del profesorado universitario en once campos científicos que recogen las áreas de conocimiento existentes, definiéndose en cada uno de ellos unos criterios específicos de evaluación acordes con las particularidades de las diferentes disciplinas científicas. Cada campo científico cuenta con un comité asesor formado por vocales con al menos tres sexenios de investigación que, en principio, deben corresponderse con el área investigadora de los solicitantes.

tabla 1. Campos Científicos

\begin{tabular}{|l|}
\hline 1. Matemáticas y Física \\
\hline 2. Química \\
\hline 3. Biología Celular y Molecular \\
\hline 4. Ciencias Biomédicas \\
\hline 5. Ciencias de la Naturaleza \\
\hline 6. Ingenierías y Arquitectura \\
\hline 7. Ciencias Sociales, Políticas, del Comportamiento y de la Educación \\
\hline 8. Ciencias Económicas y Empresariales \\
\hline 9. Derecho y Jurisprudencia \\
\hline 10. Historia y Arte \\
\hline 11. Filosofía, Filología y Lingüística \\
\hline
\end{tabular}

Según establece la Orden de 2 de diciembre de 1994, para realizar la evaluación, el solicitante seleccionará, hasta un máximo de cinco, las aportaciones que considere más destacadas para el período de seis años objeto de evaluación. Para otorgar una valoración positiva es preciso que las aportaciones presentadas contribuyan al progreso del conocimiento, la innovación y la creatividad, primando los trabajos que sean formalmente científicos, asignando un valor accesorio a las aportaciones de carácter descriptivo, referidas a la aplicación de conocimientos y los estudios divulgativos.

Los resultados de la distribución global de la evaluación por campos científicos (19892005) es la recogida en la Gráfico 1 (CNEAI, 2005).

En el Gráfico 1 se observan los malos resultados obtenidos en el Campo 8, Ciencias Económicas y Empresariales, que con un $58 \%$ de sexenios concedidos sobre solicitados está muy por debajo de la media, el 71\%. Estas diferencias son todavía más acentuadas si se analiza la composición de las distintas áreas de conocimiento que integran los distintos campos científicos, señalando el área de "Economía Financiera y Contabilidad" con tan sólo $51 \%$ de sexenios concedidos sobre los solicitados, siendo además una de las áreas que peor resultados obtiene en términos porcentuales entre las casi 200 áreas de conocimiento que configuran los 11 campos científicos evaluados (CNEAI 2005). 
GRÁFICO 1: RESULTADO dE LA EVALUACIÓN DE LOS SEXENIOS POR CAMPOS DE CONOCIMIENTO EN EL PERÍODO 1989-2005. UNIVERSIDADES PÚBLICAS

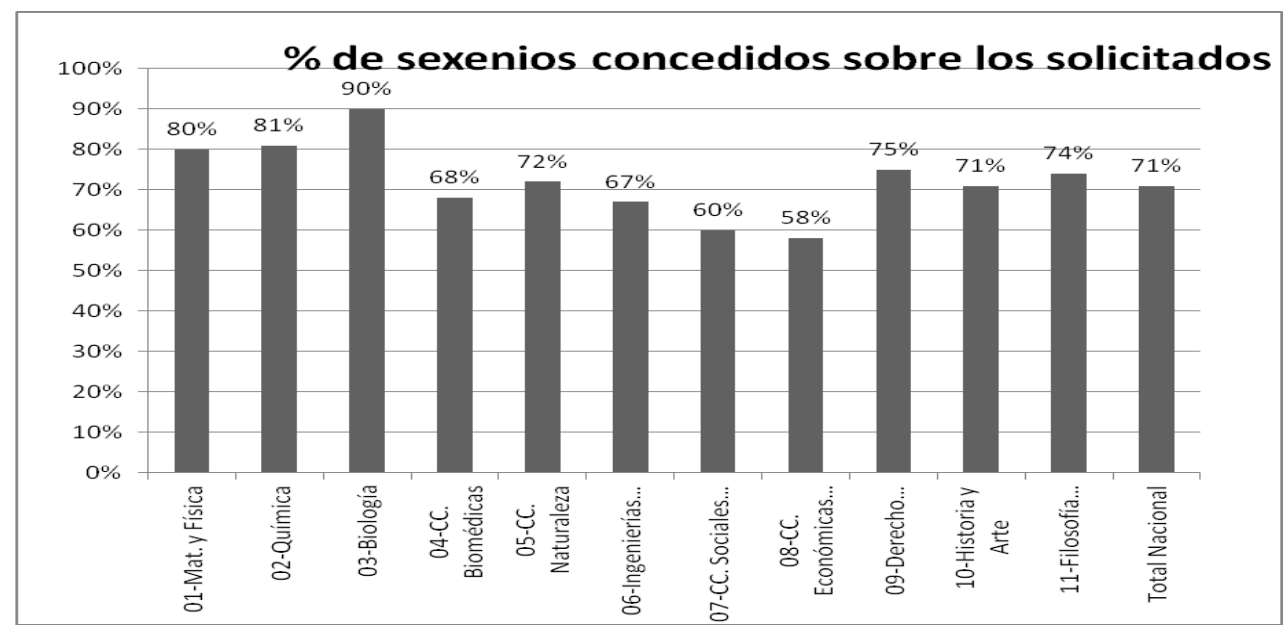

Fuente: CNEAI.

Esta distribución condiciona significativamente la participación del profesorado de cada Campo Científico en las diferentes facetas universitarias vinculadas con la posesión de sexenios. Expuesta esta situación, para profundizar en el análisis de cómo están distribuidos los sexenios, se utilizará la información correspondiente al número de profesores que pueden formar parte de las comisiones de acceso a los cuerpos docentes universitarios. Recordar que se exige a los CU tener reconocidos al menos dos sexenios de investigación, y a los TU, CEU y TEU doctores un sexenio. Ahora bien, debido a que la acreditación en el Campo 8 únicamente puede ser de CU y TU, que el número de CEU con respecto al resto de cuerpos de profesores universitarios es poco significativo y que los CEU y TEU que poseen un sexenio de investigación son pocos, nuestro estudio se circunscribirá únicamente a los cuerpos docentes, CU y TU.

Dado que los sexenios exigidos a los componentes de las comisiones de habilitación y acceso son idénticos, se ha tomando como referencia la lista definitiva de sorteables para formar parte de las comisiones de habilitación en su convocatoria de septiembre de 2005, publicada por la Secretaría General del Consejo de Coordinación Universitaria, y el listado por áreas de conocimiento del profesorado en servicio activo publicado por la citada Secretaría (2004), la Tabla 2 muestra el porcentaje de miembros de cada campo que pueden formar parte de las mismas, por categoría, con respecto al número total de profesores existente en cada uno de ellos.

Como puede observarse, el porcentaje de CU y TU del Campo 8 con respecto a la media es menor, respectivamente, en $27,03 \%$ y un $26,67 \%$. Esta situación afecta de forma negativa al número de profesores que, en dicho campo, pueden participar en las anteriormente mencionadas facetas de la vida universitaria, entre ellas formar parte de las comisiones de acceso. 
TABLA 2. PORCENTAJE DE PROFESORES CU Y TU QUE PUEDEN FORMAR PARTE DE LAS COMISIONES DE ACCESO POR CATEGORÍA Y CAMPO CIENTÍFICO.

\begin{tabular}{|l|c|c|}
\cline { 2 - 3 } \multicolumn{1}{l|}{} & \multicolumn{2}{c|}{$\%$ Categoría } \\
\hline Campo Científico & TU \\
\hline 1. Matemáticas y Física & $83,20 \%$ & $75,50 \%$ \\
\hline 2. Química & $97,58 \%$ & $87,81 \%$ \\
\hline 3. Biología Celular y Molecular & $98,79 \%$ & $90,95 \%$ \\
\hline 4. Ciencias Biomédicas & $79,50 \%$ & $64,66 \%$ \\
\hline 5. Ciencias de la Naturaleza & $86,80 \%$ & $79,18 \%$ \\
\hline 6. Ingenierías y Arquitectura & $67,90 \%$ & $61,89 \%$ \\
\hline 7. Ciencias Sociales, Políticas, del Comportamiento y de la Educación & $87,56 \%$ & $55,33 \%$ \\
\hline 8. Ciencias Económicas y Empresariales & $53,70 \%$ & $40,36 \%$ \\
\hline 9. Derecho y Jurisprudencia & $89,05 \%$ & $73,36 \%$ \\
\hline 10. Historia y Arte & $85,49 \%$ & $69,85 \%$ \\
\hline 11. Filosofía, Filología y Lingüística & $\mathbf{8 0 , 7 3 \%}$ & $\mathbf{6 7 , 0 3 \%}$ \\
\hline Media & $73 \%, 31 \%$ \\
\hline
\end{tabular}

Fuente: Elaboración propia

\section{LA EVALUACIÓN DE LA ACTIVIDAD INVESTIGADORA DEL PROFESORADO UNIVERSITARIO EN EL CAMPO 8}

Expuesta la situación general de los profesores que pueden formar parte de las comisiones de acceso en los once campos científicos, a continuación nuestro estudio se centrará en las áreas de conocimiento que componen el Campo 8, entre las que se encuentra "Economía Financiera y Contabilidad". Utilizando la información de la CNEAI (2002; 2005) y de la Secretaría General de Coordinación Universitaria del epígrafe anterior, en la Tabla 3 se muestra el porcentaje de profesores para estas categorías, que en 2002, 2003 y 2004 pueden formar parte de las comisiones de acceso.

Si comparamos los porcentajes del Economía Financiera y Contabilidad con respecto a la media del campo científico 8, los porcentajes son menores en todos los años para los CU, en un $18,34 \%, 18,64 \%$ y $18,48 \%$, respectivamente. De forma idéntica, para los TU, el porcentaje también es menor, en un $14,98 \%, 19,56 \%$ y $17,15 \%$. 
TABLA 3. PORCENTAJE DE PROFESORES QUE PUEDEN Formar PARTE DE LAS COMISIONES DE ACCESO POR CATEGORÍA Y CAMPO CIENTÍFICO

\begin{tabular}{|c|c|c|c|c|c|c|}
\hline & \multicolumn{6}{|c|}{ Campo 8. Ciencias Económicas y Empresariales } \\
\hline & \multicolumn{3}{|c|}{ CU } & \multicolumn{3}{|c|}{ TU } \\
\hline & 2002 & 2003 & 2004 & 2002 & 2003 & 2004 \\
\hline Análisis Geográfico Regional & $76,92 \%$ & $88,37 \%$ & $86,05 \%$ & $54,61 \%$ & $48,08 \%$ & $50,00 \%$ \\
\hline $\begin{array}{l}\text { Comercialización e Investigación de } \\
\text { Mercados }\end{array}$ & $30,77 \%$ & $38,46 \%$ & $38,46 \%$ & $20,00 \%$ & $31,20 \%$ & $33,60 \%$ \\
\hline Economía Aplicada & $40,96 \%$ & $49,83 \%$ & $47,18 \%$ & $28,55 \%$ & $32,13 \%$ & $33,50 \%$ \\
\hline Economía Financiera y Contabilidad & $28,86 \%$ & $36,48 \%$ & $35,22 \%$ & $19,00 \%$ & $17,51 \%$ & $23,21 \%$ \\
\hline Economía, Sociología y Política Agraria & $61,54 \%$ & $68,00 \%$ & $60,00 \%$ & $57,78 \%$ & $57,69 \%$ & $44,23 \%$ \\
\hline Fundamentos del Análisis Económico & $63,39 \%$ & $76,79 \%$ & $74,11 \%$ & $43,64 \%$ & $65,11 \%$ & $66,36 \%$ \\
\hline Geografía Humana & $80,85 \%$ & $83,64 \%$ & $81,82 \%$ & $63,82 \%$ & $59,01 \%$ & $61,49 \%$ \\
\hline Historia e Instituciones Económicas & $72,13 \%$ & $77,78 \%$ & $82,54 \%$ & $66,38 \%$ & $73,08 \%$ & $69,23 \%$ \\
\hline Organización de Empresas & $35,78 \%$ & $38,84 \%$ & $39,67 \%$ & $17,27 \%$ & $20,06 \%$ & $32,77 \%$ \\
\hline Media & $47,20 \%$ & $55,12 \%$ & $53,70 \%$ & $33,98 \%$ & $37,07 \%$ & $40,36 \%$ \\
\hline
\end{tabular}

Fuente: Elaboración propia

Como puede observarse las diferencias son notables tanto en CU como en TU, de lo que se desprende que las evaluaciones realizadas por el comité asesor del Campo 8, merecidamente $\mathrm{O}$ no, han afectado negativamente a determinadas áreas de conocimiento, entre las que destaca "Economía Financiera y Contabilidad". En efecto, dicha área obtiene unos porcentajes significativamente inferiores a la media del Campo 8, y muy inferiores al conjunto de campos científicos. Si la comparación se hubiera realizado respecto al conjunto de campos científicos, para el año 2004 los porcentajes son menores en un $45,51 \%$ para CU y $43,82 \%$ para TU.

No parece lógico el resultado que presentan determinadas áreas económicas, máxime si se compara con los resultados de otros campos científicos. Dando un margen de confianza a la investigación realizada en "Economía Financiera y Contabilidad", tal vez el origen del problema se encuentre, al menos en parte, en las evaluaciones efectuadas por los miembros de los comités asesores del Campo 8.

Siguiendo con esta reflexión sería interesante analizar si existe correlación entre el número de miembros de los comités asesores, según el área de conocimiento a la que pertenecen, y el porcentaje de profesores TU y CU que, al haber obtenido los sexenios precisos, pueden integrar las comisiones de acceso, en comparación con el número total de profesores de un área concreta. 
Utilizando el listado por áreas de conocimiento del profesorado en servicio activo publicado por la Secretaría General del Consejo de Coordinación Universitaria (2004) y las resoluciones hasta 2005 donde se nombran los miembros de los diferentes comités encargados de asesorar a la CNEAl, en la Tabla 4 se muestra el número de profesores del Campo 8 por área de conocimiento presentes en las dieciséis evaluaciones realizadas en comparación con el numero de CU y TU que componen el área de conocimiento.

tabla 4. Número de participaciones en las Comisiones del Grupo 8 por Área de CONOCimiento y el total de CU Y TU POR ÁREA

\begin{tabular}{|c|c|c|c|c|c|c|}
\hline \multirow{2}{*}{ Etiqueta } & \multirow{2}{*}{ Áreas de Conocimiento } & \multirow{2}{*}{$\begin{array}{c}\mathrm{N}^{\circ} \text { de } \\
\text { participacion } \\
\text { es }\end{array}$} & \multicolumn{2}{|c|}{$\mathrm{CU}$} & \multicolumn{2}{|c|}{ TU } \\
\hline & & & Totales & Orden & Totales & Orden \\
\hline AGR & Análisis Geográfico Regional & 8 & 43 & 7 & 156 & 6 \\
\hline $\mathrm{CIV}$ & $\begin{array}{l}\text { Comercialización e Investigación de } \\
\text { Mercados }\end{array}$ & 4 & 39 & 8 & 125 & 8 \\
\hline EA & Economía Aplicada & 20 & 301 & 1 & 806 & 1 \\
\hline EFC & Economía Financiera y Contabilidad & 7 & 159 & 2 & 474 & 2 \\
\hline ESPA & Economía, Sociología y Política Agraria & 0 & 25 & 9 & 52 & 9 \\
\hline FAE & Fundamentos del Análisis Económico & 14 & 112 & 4 & 321 & 4 \\
\hline $\mathrm{GH}$ & Geografía Humana & 8 & 55 & 6 & 161 & 5 \\
\hline HIE & Historia e Instituciones Económicas & 14 & 63 & 5 & 130 & 7 \\
\hline OE & Organización de Empresas & 8 & 121 & 3 & 354 & 3 \\
\hline
\end{tabular}

Fuente: Elaboración propia

De donde se desprende que determinadas áreas, como "Economía Aplicada", ha tenido más representantes que convocatorias evaluadoras, y áreas como "Historia e Instituciones Económicas" y "Fundamentos del Análisis Económico", han tenido una presencia en las comisiones de evaluación de la CENAI muy superior a otras como "Economía Financiera y Contabilidad" que tienen mucho más peso en número de Catedráticos y Titulares que forman el área.

Los resultados indican que la composición de las comisiones evaluadoras depende, en gran medida, del número de profesores del área con los sexenios requeridos, con independencia del peso real de dicha área en cuanto a número de profesores, peticiones, etc. Por ello, áreas como Economía Financiera y Contabilidad, que es la segunda del grupo en número de CU y TU, sin embargo, el porcentaje de profesores en posición de formar parte de las comisiones es muy reducido. Esta circunstancia conlleva una escasa representación en las mismas y, por ello, podría influenciar, a su vez, resultados muy bajos en los sexenios reconocidos, siendo muy difícil romper este círculo vicioso.

La cuestión es si esta situación ha podido afectar al número de profesores por área de conocimiento del Campo 8 que pueden formar parte de las comisiones de acceso. Para responder esta cuestión, se analiza la correlación lineal existente entre los datos de 2004 de la Tabla 3 y la Tabla 4. Se calcula el coeficiente de correlación de Pearson 
y se efectúa un contraste de la X2 para ver la significación de dicha correlación entre las variables seleccionadas, siendo los resultados los que se muestra en la Tabla 5.

TABla 5. CorRelación ENTRE PARTICIPACIÓN Y \% CU Y TU QUE PUEDen FORMAR PARTE DE LAS COMISIONES DE ACCESO

\begin{tabular}{|c|c|c|c|c|}
\hline & \\
\hline & & Participación & $\% \mathrm{CU}$ & $\%$ TU \\
\hline \multirow[b]{2}{*}{ Todas las áreas } & Participación & $\begin{array}{l}\text { Correlación de Pearson } \\
\text { Sig. (bilateral) } \\
\text { N }\end{array}$ & $\begin{array}{c}0,175 \\
0,652 \\
9\end{array}$ & $\begin{array}{c}0,249 \\
0,518 \\
9\end{array}$ \\
\hline & $\% \mathrm{CU}$ & $\begin{array}{l}\text { Correlación de Pearson } \\
\text { Sig. (bilateral) } \\
\text { N }\end{array}$ & & $\begin{array}{c}0,899^{* *} \\
0,001 \\
9\end{array}$ \\
\hline \multirow{2}{*}{$\begin{array}{l}\text { Excluyendo las } \\
\text { áreas de EA y } \\
\text { ESPA }\end{array}$} & Participación & $\begin{array}{l}\text { Correlación de Pearson } \\
\text { Sig. (bilateral) } \\
\text { N }\end{array}$ & $\begin{array}{c}0,606 \\
0,149 \\
7\end{array}$ & $\begin{array}{c}0,783^{*} \\
0,037 \\
7\end{array}$ \\
\hline & $\% \mathrm{CU}$ & $\begin{array}{l}\text { Correlación de Pearson } \\
\text { Sig. (bilateral) } \\
\text { N }\end{array}$ & & $\begin{array}{c}0,894^{*} \\
0,007 \\
7\end{array}$ \\
\hline
\end{tabular}

* La correlación es significativa al nivel 0,05 (bilateral).

** La correlación es significativa al nivel 0,01 (bilateral).

Como puede observarse en fila "Todas las áreas" de la Tabla 5, se desprende que no hay una correlación significativa entre "Participación" y "\% CU" ni tampoco entre "Participación" y "\% TU".

Si se eliminaran las áreas de "Economía Aplicada" y "Economía, Sociología y Política Agraria", cuyas participaciones en los comités asesores tiene valores atípicos respecto a la media y se repiten los cálculos sobre el coeficiente, se muestra que aunque no hay una correlación significativa entre "Participación" y "\% CU", si que la hay entre "Participación" y "\% TU". En consecuencia, se podría concluir que, si se excluyen las áreas con un número de representantes que distorsionan la media, la presencia de miembros de un área determinada en los comités asesores es un factor determinante para que los TU de dichas áreas obtengan los sexenios requeridos para formar parte de las comisiones de acceso.

Si bien los resultados obtenidos hasta ahora por si sólo ya nos deberían hacer reflexionar sobre la composición de las comisiones de evaluación de la CNEAl, dichos resultados son todavía más alarmantes si se consideran de forma independiente la subárea "Contabilidad" de la subárea "Economía Financiera". Para ello, tomando como referencia el profesorado del área en servicio activo publicado por Secretaría General del Consejo de Coordinación Universitaria (2004), y la lista definitiva de sorteables en la convocatoria de habilitación de 21 de septiembre de 2005, se procedió a clasificar a cada profesor al subárea de Economía Financiera o 
Contabilidad, según la disciplina con la que tuviera una mayor vinculación atendiendo a su labor docente, producción investigadora y actividad profesional.

En la Tabla 6 se muestra el porcentaje de profesores por categoría que en el año 2004 pueden formar parte de las comisiones de acceso en "Economía Financiera" y en "Contabilidad".

TABLA 6. \% DE CU Y TU QUE PUEDEN formaR PARTE DE LAS COMISIONES DE ACCESO EN "ECONOMía FINANCIERA" Y EN "CONTABILIDAD"

\begin{tabular}{|l|c|c|c|c|}
\cline { 2 - 5 } \multicolumn{1}{c|}{} & \multicolumn{2}{c|}{ Economía Financiera } & \multicolumn{2}{c|}{ Contabilidad } \\
\cline { 2 - 5 } \multicolumn{1}{c|}{} & CU & TU & CU & TU \\
\hline \% Profesores Comisiones Acceso & $46,05 \%$ & $30,57 \%$ & $25,30 \%$ & $16,33 \%$ \\
\hline $\begin{array}{l}\text { Diferencia Total Campos } \\
\text { Científicos }\end{array}$ & $-34,68 \%$ & $-36,46 \%$ & $-55,43 \%$ & $-50,70 \%$ \\
\hline Diferencia Campo 8 & $-7,65 \%$ & $-9,80 \%$ & $-28,40 \%$ & $-24,04 \%$ \\
\hline Diferencia EFyC & $10,83 \%$ & $7,36 \%$ & $-9,92 \%$ & $-6,88 \%$ \\
\hline
\end{tabular}

Tal y como se muestra en la Tabla 6, dentro de "Economía Financiera y Contabilidad", área cuyos resultados en comparación al total de Campos Científicos y con el conjunto del Campo 8 ya son de por si bastante malos, la subárea "Contabilidad" es la que peor resultados presenta. Es decir, en "Contabilidad" es donde se da un menor porcentaje tanto de CU como de TU que, al disponer de los sexenios requeridos, pueden formar parte de las comisiones de acceso.

En la Tabla 7 se repite el análisis de correlación efectuado, separando la subárea "Contabilidad" de "Economía Financiera" y diferenciando dentro de los 7 participantes del área en la comisión del Campo 8, que únicamente 2 son profesores de "Contabilidad" y que los otros 5 profesores son de "Economía Financiera".

Eliminando las áreas con valores atípicos, se puede observar que hay una correlación significativa entre "Participación" y "\% CU", así como "\% TU". Es decir, se constata la notable influencia que tienen el número de miembros de un área determinada en las comisiones de evaluación, respecto al porcentaje de profesores de dicha área con los sexenios necesarios para acceder a las comisiones.

Como señala Sanz (2004), la transparencia es un valor esencial de la evaluación de la investigación, y la selección de las personas que la lleven a cabo es un elemento decisivo. Si bien no hay porqué dudar de la transparencia del proceso de selección de los responsables de las comisiones de evaluación de la CNEAI, si convendría reflexionar sobre su idoneidad a la hora de evaluar el trabajo realizado en áreas de conocimiento distintas a las suyas. Especialmente, si, como señala Lee (1997), existen ciertas élites que ejercen una gran influencia en buena parte de las principales revistas internacionales, por lo que la publicación de algunos trabajos no tiene porqué estar necesariamente ligado a la mayor o menor calidad de los mismos, sino a otros factores entre los que estaría la oportunidad de la investigación realizada. 
TABLA 7. CoRRELACIÓN ENTRE PARTICIPACIÓN Y \% CU Y TU QUE PUEDEN FORMAR PARTE DE LAS COMISIONES dE ACCESO (DIFERENCIANDO ECONOMÍA FINANCIERA DE CONTABILIDAD)

\begin{tabular}{|c|c|c|c|c|}
\hline & Participación & $\% \mathrm{CU}$ & $\%$ TU \\
\hline \multirow[t]{2}{*}{ Todas las áreas } & Participación & $\begin{array}{l}\text { Correlación de Pearson } \\
\text { Sig. (bilateral) } \\
\mathrm{N}\end{array}$ & $\begin{array}{c}0,341 \\
0,336 \\
10\end{array}$ & $\begin{array}{c}0,419 \\
0,229 \\
10\end{array}$ \\
\hline & $\%$ CU & $\begin{array}{l}\text { Correlación de Pearson } \\
\text { Sig. (bilateral) } \\
\text { N }\end{array}$ & & $\begin{array}{c}0,916^{* *} \\
0,000 \\
10\end{array}$ \\
\hline \multirow[t]{2}{*}{$\begin{array}{l}\text { Excluyendo las } \\
\text { áreas de EA y } \\
\text { ESPA }\end{array}$} & Participación & $\begin{array}{l}\text { Correlación de Pearson } \\
\text { Sig. (bilateral) } \\
N\end{array}$ & $\begin{array}{c}0,751^{*} \\
0,032 \\
8\end{array}$ & $\begin{array}{c}0,901^{* *} \\
0,002 \\
8\end{array}$ \\
\hline & $\%$ CU & $\begin{array}{l}\text { Correlación de Pearson } \\
\text { Sig. (bilateral) } \\
\text { N }\end{array}$ & & $\begin{array}{c}0,914^{* *} \\
0,001 \\
8\end{array}$ \\
\hline
\end{tabular}

* La correlación es significativa al nivel 0,05 (bilateral).

** La correlación es significativa al nivel 0,01 (bilateral).

Dejando al margen otros factores, podemos concluir que la mínima representación de los profesores de "Contabilidad" en los comités asesores de la CNEAl, conlleva unos peores resultados en la evaluación de los profesores del área, en la medida en que no hay expertos en la materia que evalúen la investigación, atendiendo a las características particulares de la Contabilidad. Esto termina cerrando un círculo vicioso, ya que si no hay CU y TU con sexenios no pueden formar parte de las comisiones de expertos que los otorgan.

\section{UNA REFLEXIÓN EN FUNCIÓN DE LAS TEORÍAS DE LA MOTIVACIÓN}

Como consecuencia de lo hasta ahora apuntado, es preciso destacar el efecto desmotivador que sobre los profesores de contabilidad puede tener tanto del proceso seguido en la evaluación de los sexenios de investigación, como fundamentalmente los resultados finales. El reconocimiento de un sexenio de investigación es el reconocimiento de un trabajo realizado durante un periodo extenso de tiempo, por lo que puede y debe ser analizado a la luz de las teorías de motivación.

La "Teoría de la equidad" analiza el efecto que produce sobre la motivación la capacidad de las personas para efectuar comparaciones con el ambiente interno y externo que lo rodea. El autor más destacado de esta teoría es Adams (1963; 1965) quién considera que a las personas además de interesarles la obtención de recompensas por su desempeño, también desean que estas sean equitativas, lo que transforma en más compleja la motivación. Es decir, existe una tendencia a comparar el esfuerzo realizado con las recompensas obtenidas, realizándose además 
comparaciones de la relación esfuerzo recompensa que reciben otras personas. Si estamos recibiendo lo mismo que los demás nos sentimos satisfechos y motivados para seguir adelante, de lo contrario nos desmotivamos. Evidentemente y bajo el prisma de esta teoría, a poco que se analicen los resultados de las evaluaciones obtenidas por los profesores de contabilidad, especialmente si se comparan con otras áreas de investigación, es fácil deducir el efecto desmotivador conseguido.

Todo esto todavía se acentúa si introducimos en nuestro análisis la denominada "Teoría de las expectativas". Esta teoría, cuyos autores más destacados son Vroom (1964) y Porter y Lawler (1968), sostiene que las personas tienen creencias y abrigan esperanzas y expectativas respeto a los sucesos futuros de sus vidas. Las personas altamente motivadas son aquellas que perciben ciertas metas e incentivos como valiosos para ellos y, a la vez, perciben subjetivamente que la probabilidad de alcanzarlos es alta. De esta forma la fuerza de la motivación de una persona en una situación determinada equivale al producto entre el valor que la persona le asigna a la recompensa y la expectativa de su posible logro. Tras analizar la situación en España los profesores de contabilidad, vemos que el esfuerzo realizado en la investigación contable, en la mayor parte de los casos, no se traduce en un desempeño, reconocimiento de sexenios, lo que puede ser causa de desmotivación en el profesor que no ve recompensado su trabajo.

Para finalizar, la "Teoría de las Metas" (Locke, 1969) indica que una de las premisas básicas para que el individuo desarrolle un compromiso con la consecución de los objetivos es que éstos sean específicos, y que reciba un feedback para poder potenciar al máximo los logros (Becker, 1978), algo que, a la vista de las resoluciones de la CNEAl, no siempre ocurre.

La actualidad y la cada vez mayor relevancia que tiene el reconocimiento de la investigación hacen más necesario que nunca, que se aborde esta situación cuestión desde diferentes perspectivas, de ahí el interés que puede tener introducir en nuestro análisis las Teorías de Motivación. No obstante ello no debe desviarnos del principal objetivo que nos marcamos al iniciar el presente trabajo, es decir, reflexionar sobre la incidencia que la composición de las comisiones de evaluación de la CNEAl tiene sobre el porcentaje de profesores que pueden formar parte de las comisiones de acceso o dicho de otro modo, de los sexenios que se conceden a las áreas dependiendo de su representación. La situación creada va a suponer, como consecuencia más visible e inmediata, que el grupo de profesores que en "Economía Financiera y Contabilidad" pueden formar parte de las comisiones de acceso queda reducida a una "elite".

\section{DISCUSIÓN DE LOS RESULTADOS}

La normativa ha ido paulatinamente otorgando al sexenio un papel más importante en las diferentes actividades del profesorado universitario, condicionando su retribución, promoción, dedicación docente, actividad investigadora, así como formar parte de las comisiones de acreditación y acceso a los cuerpos docentes universitarios. Situación que tiene visos de agravarse en los aspectos ya contemplados, y extenderse a áreas como ocupar cargos académicos, gobernanza, contratación, selección, financiación, prestigio y calidad de la universidad.

Este escenario otorga el control de la universidad a las élites que tengan reconocidos los sexenios exigidos. La gestión, contratación, órganos decisorios y de representación, disponibilidad de recursos económicos, promoción y evaluación de la actividad investigadora, está reservada a un grupo reducido de profesores. Las divergencias existentes en los sexenios concedidos a cada área de conocimiento crean desigualdades en las oportunidades de participación en la vida universitaria. 
Divergencias que también afectan en la docencia y la investigación. Advertir que aumentar la carga docente de los profesores sin sexenios supone disponer de menos tiempo para poder investigar, redundando en que se destinen los esfuerzos a investigar para alcanzar el codiciado sexenio, pudiendo abandonar o descuidar nuestras labores docentes.

Tomando como referencia la información resultante del trabajo, en esta situación de inferioridad se encuentra el Campo 8. Ciencias Económicas y Empresariales, situación que se agrava en el área de conocimiento "Economía Financiera y Contabilidad" al presentar unos porcentajes menores en comparación con el conjunto del campo. Y, lógicamente, como profesores de contabilidad, no podemos evitar interesarnos por el estado en la cual se encuentra nuestra disciplina, pudiendo comprobar cómo dicha situación de desventaja se encuentra aún más acentuada.

Según se desprende del presente estudio, en la valoración de la investigación contable influye negativamente la escasa presencia que hasta el momento los profesores de dicha subárea han tenido en las citadas comisiones de evaluación, y que esta mínima representación conlleva unos peores resultados en la evaluación de los profesores del subárea contable.

Aunque se pudiera admitir que en contabilidad vamos con cierto retraso en la internacionalización (García y Laínez-Gadea, 2004), no hay porqué dudar de la calidad e interés de la investigación desarrollada en esta disciplina, y conviene reflexionar detenidamente sobre el porqué de la situación planteada. Evidentemente, el hecho de que denunciemos esta situación no quiere decir que éste sea el único factor que incide en los malos resultados que presenta la disciplina contable. Ni tampoco quiere decir que los miembros de las comisiones de evaluación intencionadamente quieran favorecer a profesores de sus áreas. Nada más lejos de nuestra intención. No obstante, pensamos que podría poner de manifiesto que no contar con un experto en contabilidad supone que no se tengan en cuenta las peculiaridades de dicha disciplina, se apliquen los criterios de áreas que no se corresponden con la investigación en contabilidad, y no se determine qué investigación debe considerarse relevante para ser valorada positivamente.

Evaluar la investigación contable con los criterios de las ciencias puras supone que su interés sea académicamente aceptable pero que carezca de fundamento. Circunstancia que explicaría por qué la investigación contable fracasa a la hora de mejorar la práctica contable (Inanga, 2005). Dados los escasos incentivos que el sistema de evaluación asigna a la investigación centrada en la práctica, está conduciendo al error de ser dirigida a los académicos en lugar de a los profesionales (Evans et al., 2011). Se demuestra una estrechez de miras en la evaluación de la disciplina contable, si se toma como referente revistas listadas en el Journal of Citations Report, si se penaliza la coautoría y la publicación en revistas que no son del área, y no se tiene en cuenta la investigación publicada en revistas de carácter profesional, monografías o manuales (Lizcano, 2012; Adler y Harzing, 2009).

Efectuar una evaluación adecuada de la actividad investigadora implica tener en consideración las particularidades de cada área de conocimiento. Y en el caso de contabilidad, la aplicación de unos criterios generales no responde al hecho diferencial de la investigación en contabilidad. La vinculación de nuestra disciplina con el ámbito empresarial y profesional, supone que esta faceta debería tenerse más en cuenta, puesto que no tiene sentido el actual enfoque de la investigación contable centrado en el impacto en lugar del rigor puramente metodológico, y el establecimiento de una interacción más significativa entre los profesionales y los docentes de contabilidad (Diamond, 2005). El abandono de la de la realidad, Shapiro 2005), que se ha producido a causa de la revolución empírica en contabilidad, debe 
abandonarse en favor de un enfoque más orientado a los problemas de la investigación contable y de la práctica (Williams, 2009).

La cantidad es el criterio más simple y el único que no presenta, en principio, dudas, al poder ser medido numéricamente sin grandes problemas, no obstante no siempre resulta sencilla la medición del impacto, y especialmente de la importancia o la calidad. Para medir el impacto se suele recurrir a estudios bibliométricos (Bordons y Zulveta, 1999; Bordons y Gómez, 1997; Osca, 2000; Hicks, 1999; Schomch, 1997; Sanz y Martín, 1997), pero la importancia y sobretodo la calidad de un trabajo de investigación no puede ser capturada exclusivamente con indicadores bibliográficos, exige de la realización de juicios de valor por parte de expertos en la materia. En este sentido Johnes y Johnes (1995) advierten de la gran dificultad que encierra construir una definición operativa, objetiva e indiscutible de calidad de la investigación. Dificultad que se verá acrecentada si los responsables de construir dicha definición ni tan siquiera son expertos en el tema.

La consecuencia última de la situación planteada es el efecto desmotivador que sobre los profesores de contabilidad puede tener tanto del proceso seguido en la evaluación de los sexenios de investigación, como los resultados finales del mismo. El reconocimiento de un sexenio de investigación es el reconocimiento de un trabajo realizado durante un periodo extenso de tiempo, y por poco que se analicen los resultados de las evaluaciones obtenidas por los profesores de contabilidad, especialmente si se comparan con otras áreas de investigación, es fácil deducir el efecto desmotivador conseguido. El esfuerzo realizado en la mayor parte de los casos no se traduce en un desempeño, lo que puede ser causa de desmotivación en el profesor que no ve recompensado su trabajo. Desmotivación que se agrava si se añade el hecho de que la evaluación de la CNEAl se efectúa a posteriori, sin justificar sus decisiones y no detalla claramente los criterios utilizados, que además pueden cambiar de una evaluación a otra. Destacar al respecto el trabajo de Buela-Casal (2007), quién reflexiona sobre la ausencia de criterios claros de evaluación de la actividad del profesorado y la subjetividad imperante en los procesos.

En definitiva, a tenor de lo expuesto hasta ahora, no es de extrañar que muchos profesores universitarios de contabilidad puedan sentirse desmotivados, frustrados, e incluso desmoralizados al ver el escaso reconocimiento que en su propio país tiene la investigación que realizan, como muestran los resultados de Arquero y Donoso (2013), en los que se demuestra el incremento tanto de los niveles de SQT, (Síndrome del Quemado por el Trabajo), como del abandono de buena parte del profesorado joven, que constituye el futuro de la plantilla docente e investigadora en contabilidad.

\section{BIBLIOGRAFÍA}

ADAMS, J.S. [1963]: Toward an understanding of inequity, Journal of Abnormal and Social Psychology, 67: 422-436.

ADAMS, J.S. [1965]: Inequity in social exchange, En Leonard Berkowitz, Advances in Experimental Social Psychology, 2, Academic Press, New York.

AdLeR, N.J. y HARZING, A. [2009]: When Knowledge Wins: Transcending the Sense and Nonsense of Academic Rankings, Academy of Management Learning \& Education, 8 (1), 72-95.

ARnOld, T., BUtLer, A., CRACK, T. y ALtintig, A. [2003]: Impact: what influences finance research?, Journal of Business, 76 (2), 343-361.

ARQUERO, J.L Y DONOSO, J.A. [2013]: Síndrome del quemado en profesores universitarios de contabilidad. Estudio de los niveles asociados a las tareas docentes e investigadoras. Revista de Contabilidad. Pendiente de publicación. 
BauWens, L., Kirman, A., Lubrano, M. y Protopopescu, C. [2002]: Ranking European Economic Departments: a Statistical Approach, Mimeo, CORE, Université Catholique de Louvain.

BECKER, L. J. [1978]: Joint effect of feedback and goal setting on performance. A field study of residential energy conservation, Journal of Applied Psychology, 63, 428-433.

Bergantiños, G., DA Rocha, J.M. y Palomé, PH. [2002]: La Investigación Española en Economía, 1995-1999, Investigaciones Económicas, 26 (2), 373-392.

BORDONS, M. y GÓMEZ, I. [1997]: La actividad científica española a través de indicadores bibliométricos en el periodo 1990-93, Revista General de Información y Documentación, 2, 69-86.

BORDONS, M. y ZULUETA, M.A. [1999]: Evaluación de la actividad científica a través de indicadores bibliométricos, Revista Española de Cardiología, 52, 798-800.

Buela-CASAL, G. [2007]: Reflexiones sobre el sistema de acreditación del profesorado funcionario de Universidad en España, Psicothema, 19-3, 473-482.

CAMPANARIO, J.M. [2002]: El sistema de revisión por expertos (peer review): muchos problemas y pocas soluciones, Revista Española de Documentación Científica, 3, 166-184.

Cole, S. [1998]: How Does Peer Review Work and Can It be Improved?, Minerva, 36 (20), 179-189.

COMBES. P.P. y LINNEMER, L. [2002]: Measuring and Ranking Economics Throughout Europe, Mimeo, París.

COMISIÓN NACIONAL EVAlUADORA DE LA ACTIVIDAD INVESTIGADORA (CNEAI) [2002]: Folleto Informativo, Ministerio de Educación, Cultura y Deporte,

http://www.mecd.gob.es/dctm/ministerio/horizontales/ministerio/organismos/ cneai/2009-folleto-cneai.pdf? documentld=0901e72b8009157d.

COMISIÓN NACIONAL EVALUADORA DE LA ACTIVIDAD INVESTIGADORA (CNEAI) [2005]: Memoria de los resultados de las evaluaciones realizadas de 1989 a 2005 (Profesores de Universidad), Ministerio de Educación, Cultura y Deporte, http://www.mecd.gob.es/dctm/ministerio/horizontales/ministerio/organismos/ cneai/2005-memoria-1989-2005universidad.pdf?documentld=0901e72b8008d9f6.

CoupÉ, T. [2000]: Revealed Performances Worldwide Rankings of Economists and Economics Departments, Mimeo, ECARES, Université Libre de Bruxelles.

Dahllof, U., Harris, J., Shattock, M., Staropoli, A. y Veld, R.I. [1991]: Dimensions of Evaluation: Report of the Evaluation in Higher Education, Kingsley, Londres.

De las Heras, G. y Polo, G. [2009]: La evaluación de la investigación universitaria en ciencias jurídicas en el marco general español, Revista de la Educación Superior, XXXVIII (2), 63-79.

DelGado, E. [2009]: Claroscuros en la evaluación científica en España, Jornada Medes 2009, http://ec3.ugr.es/publicaciones/BoletinMEDES10.pdf.

DIAMOND, M. [2005]: Accounting Education, Research and Practice: After Enron, Where Do We Go?, European Accounting Review, 14 (2), 353-362.

DOLADO, J.J., García, A. y ZAMORANO, G. [2001]: Rankings de investigación en economía en España: Instituciones y autores (1990-1999), Documento de Trabajo 2001-10, FEDEA.

GarcíA, M.A. y LAínez-GAdeA, J.A. [2004]: Forever destined to be extras in a Broadway show: a discussion of the status of national accounting research in an international arena, En Christopher Humphrey, Bill H.K. Lee. The Real Life Guide to Accounting Research: A Behind-the-Scenes View of Using Qualitative Research Methods, Elsevier. 73-93.

GUTHRIE, J.; BURRIT, R. y EVANS, E. [2011]: The Relationship between Academic Accounting Research and Professional Practice, En Elaine Evans, Roger Burritt and James 
Guthrie. Bridging the Gap between Academic Accounting Research and Professional Practice, The Institute of Chartered Accountants in Australia, 9-21.

HICKS, D. [1999]: The difficulty of achieving full coverage of international Social Science literature and the bibliometric consequences, Scientometrics, 44 (2), 193-215.

INANGA, E.L. Y SCHNEIDER, W.B. [2005]: The failure of accounting research to improve accounting practice: a problem of theory and lack of communication, Critical Perspectives on Accounting, 16, 227-248.

JIMÉNEZ, E., DE MOYA, F.D. y DELGADO, E. [2003]: The evolution of research activity in Spain: The impact of the National Commission for the Evaluation of Research Activity (CNEAl), Research Policy, 32 (1), 123-142.

JIMÉNEZ, E., ROBINSON, N. Y CABEZAS, A. [2011]: Productividad e impacto de los investigadores españoles: umbrales de referencia por áreas científicas, Revista española de Documentación Científica, 34 (4), 505-526.

JOHNES, J. Y JOHNES, G. [1995]: Research Funding and Performance in U.K. University Departments of Economics: A Frontier Analysis, Economics of Education Review, 14 (3), 301-314

LEE, T. [1997]: The editorial gatekeepers of the accounting academy, Accounting, Auditing and Accountability Journal, 10 (1), 11-30.

LEY ORGÁNICA 11/1983, de 25 de agosto, de Reforma Universitaria.

LEY ORGÁNICA 4/2007, de 12 de abril, por la que se modifica la Ley Orgánica 6/2001, de 21 de diciembre, de Universidades.

LEY ORGÁNICA 6/2001, de 21 de diciembre, de Universidades.

LIZCANO, J. [2012]: La necesaria orientación social de la investigación universitaria, El Imparcial, $\quad$ http://www.elimparcial.es/sociedad/la-necesaria-orientacionsocial-de-la-investigacion-universitaria-103398.html

LOCKE, E.A. [1969]: Purpose without consciousness: A contradiction, Psycgological Reports, 21, 991-1009.

MÉndez, A., Guardiola, E. y Bellavista, J. [1997]: Evaluación de la investigación, Centro de Investigaciones Sociológicas, Madrid.

MINISTERIO DE EDUCACIÓN [2011]: Borrador del Estatuto del PDI de las Universidades Españolas. http://portal.ucm.es/c/document_library/get_file?uvid=8ea34c5f1b51-41ec-adce-3947a100869a\&groupld=406165

ORDEN DE 2 DE DICIEMBRE DE 1994, por la que se establece el procedimiento para la evaluación de la actividad investigadora en desarrollo del Real Decreto 1086/1989, de 28 de agosto, sobre retribuciones del profesorado universitario.

OSCA, J. [2000]: El "impacto" de las publicaciones científicas, Jano, 1356, 79-80.

PORTER, L. Y LAWLER, E. [1968]: Managerial Attitudes and Perfomance, Richard D. Irwin, Hommewood, Illinois.

Real DeCRETO 1086/1989, de 28 de agosto, sobre Retribuciones del Profesorado Universitario.

REAL DECRETO 99/2011, de 28 de enero, por el que se regulan las enseñanzas oficiales de doctorado.

ReAl DeCReto-Ley 14/2012, de 20 de abril, de medidas urgentes de racionalización del gasto público en el ámbito educativo.

SANZ, E. y MARTín, C. [1997]: Técnicas bibliométricas aplicadas a los estudios de usuarios, Revista General de Información y Documentación, 7 (2), 41-68.

SANZ, L. [1995]: Research actors and the state: research evaluation and evaluation of science and technology policies in Spain, Research Evaluation, 5 (1), 79-88.

SANZ, L. [2004]: Evaluación de la investigación y sistemas de ciencia, Boletín de la Sociedad Española de Bioquímica y Biología Molecular, 140, 6-10.

$\mathrm{SCHMOCH}, \mathrm{U}$. [1997]: Indicators and the relations between science and technology, Scientometrics, 38 (1), 103-116. 
SeCRetaría General del ConSEJO de CoORdinación UniversitARIA [2004]: Listado por áreas de conocimiento del profesorado universitario en servicio activo, Ministerio de Educación, Cultura y Deporte, Madrid.

SHAPIRO, I. [2005]: The flight from reality in the human sciences. Princeton, NJ: Princeton University Press.

VIDAL, J. [1993]: El Science Citation Index y la evaluación de la investigación en las universidades, Política Científica, julio (37), 57-59.

VILLAR, A. [2004]: La evaluación de la investigación en Economía. http://merlin.fae.ua/villar/Investigacion.pdf

VROOM, V. [1964]: Work and Motivation, John Wiley and Sons, Nueva York.

WILLIAMS, P.F. [2009]: Reshaping accounting research: Living in the world in which we live, Accounting Forum, 33, 274-279. 